\title{
Fault Analysis using SFCL in the Convertor System at the Load Line
}

\author{
Suman Baghel \\ M. Tech Scholar \\ Corporate Institute of Science and Technology \\ Bhopal (MP), India \\ sumanbaghe19098@gmail.com
}

\author{
Sanjeev Jarariya \\ Associate Professor \\ Corporate Institute of Science and Technology \\ Bhopal (MP), India \\ sanjeevjarariya@gmail.com
}

\begin{abstract}
Among many renewable energy sources, solar energy is considered one of the most promising resources for large-scale electricity generation. Here propose resistive SFCL if a fault occurs in a simple low voltage $(\mathrm{LV})$ network. To assess the impact of SFCL in the power system under study, the space-time approach is used to evaluate the short-circuit current in force and spurious control strategies are suggested to achieve the goal. The results complement the feasibility of the proposed A-ACO-based rationalization control for transmission activity according to the limiting circuit and fault current analyzer. The second model of the bastard chassis concludes that the chassis with residual current limiting circuit and analyzer reduces the expansion of the residual current and prevents the voltage from dropping to zero, that no artificial and temporal innovation is used as before. Intelligence-based computer procedures further shorten the working time, which also makes the frame more efficient, as the voltage is restored to its typical value in a short time if the test frame is played for 1 second in a MATLAB climate / SIMULINK. The time taken by the ACO algorithm to restore normal operating conditions in the line was 0.197 seconds, 0.206 seconds and 0.27 seconds for LLLG, LLG and LG errors, respectively.
\end{abstract}

Keywords: LG, LLG, MMPT, NHS.

\section{INTRODUCTION}

Among numerous environmentally friendly power sources, sun oriented energy is viewed as quite possibly the most encouraging assets for huge scope power age [1]. In a few nations, including Australia, increasingly photovoltaic (PV) creation frameworks are associated with the conveyance lattice on account of solid government support. The photovoltaic market is developing quickly (30-40\%) and its cost is continually falling. Numerous nations are hoping to build the utilization of environmentally friendly power. The force gadgets interface is fundamental for associating environmentally friendly power sources to the matrix. This interface has two fundamental capacities, for example, drawing the greatest force from the photovoltaic modules $[2,3]$ and changing over the immediate current (DC) into an appropriate type of substituting current (AC) for association with the lattice. Environmentally friendly power sources, for example, sunlight based energy can't be controlled like regular energy sources, so the working states of photovoltaic inverters fluctuate contingent upon the sun oriented radiation [4]. In any case, the guidelines of utilization and the maker's datasheets just concern the full burden condition.

\section{LITERATURE REVIEW}

P. Jyothi Bhaskara Rao [5] given the consonant decrease procedure helpful for sun based force plants. The reasons for sounds are examined by relating the impacts of transformer activity. In this article the writers demonstrated the consonant decrease strategy, the Space Vector Pulse Width Modulation. The strategy is carried out in MATLAB for recreation and contrasted and sinusoidal pulse width balance.

Nishant Kumar et al. [6] presented a solitary C charge of the sensor-based greatest force point checking (MPPT) battery for photovoltaic (PV) frameworks and symphonious decrease through the typical sounds search calculation (NHS). In view of the strategy of melodic sythesis, the NHS calculation is a further developed form of the quest for concordance. His inquiry capacity is improved by utilizing the typical likelihood appropriation factor.

Nishant Kumar et al. [7] In the voltage source control (VSC) control strategy, the proposed PNKLMS control innovation is utilized, which keeps up with the nature of the organization. The presentation of NHS-based MPPT and PNKLMS based control innovation with a diminished sensor approach will be tried on a created model.

Carlos D. Fuentes et al. [8] reacted to the need to diminish the heaviness of force converters without compromising productivity and dependability. The creators recognized a 
technique to further develop execution and the capacity to fall flat over quicker with lower misfortunes, just as astounding warm properties for semiconductor gadgets in inverters.

\section{III. ОвJеCTIVE}

This thesis will target on the following main objectives:

- Design of a battery converter test system in the MATLAB / SIMULINK, which in the integrated with the grid system and should drive local loads.

- The control of the relay and coordination is based on the operation in fault analysis and the limiting circuit using of the efficient of artificial approach specific to local loads.

- The operating time reduce of the fault current elimination of the load line and quickly detect the fault current to overcome its effects.

- The framwork must be made effective and efficient when handling the error situations, which includes various types of errors such as LLLG, LLG and LG errors.

\section{Methodology}

In our work likewise we have planned a test framework having SFCL sincerely worked with an electrical switch and hand-off circuit. The different computerized reasoning based calculations are being tried to foresee the working season of the electrical switch and SFCL. This examination will talk about the different procedures embraced for its testing and afterward how the SFCL is at last introduced in an IEEE 9 transport framework for collaborated activity of different hand-off circuits.

\section{A. Resistive-type SFCL}

Superconducting state to the typical express that a superconducting material shows when the vehicle current surpasses the basic worth [3].

By and by it is a non-inductive twisting comprising of a superconducting wire or foil that is associated in corresponding to an outer shunt, which can be ohmic or inductive. In case of an issue, the outside shunt offers an exchanging way for the current and consequently lessens the Joule warming of the superconductor. An ohmic outer shunt is profitable according to the perspective of the electrical organization, since it doesn't defer the zero intersection of the flow and doesn't diminish the transient strength of the organization.

Typically, the non-inductive twisting comprises of a two-wire helical curl or a substituting game plan of flapjacks. Furthermore, an appropriately cut surface superconducting mass cylinder can be utilized to make a current way like that of a twowire curl to make a resistive SFCL.

To stay away from the danger of problem areas, the superconductor should be in touch with a regularly conductive network, or a shunt layer, (for example, with YBCO covered transmitters or BSCCO mass tubing). The capacity of the framework or the detour layer is to advance uniform proliferation of the extinguish; accordingly they have a high warm and electrical conductivity.

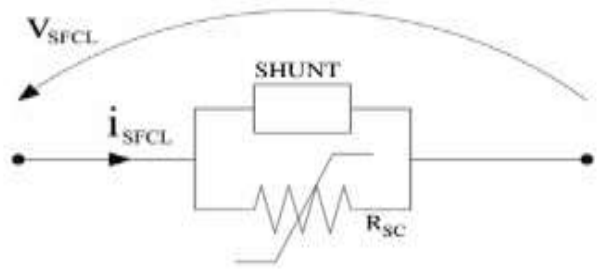

Fig. 1 Resistive-type SFCL of circuit scheme.

A circuit graph of a SFCL resistor is displayed in Figure 1. The nonlinear resistor RSC is given by what could be compared to the superconductor and the typical conductive network or shunt layer.

The best way to get a fitting incentive for the SFCL resistor is to expand the length of SC. Notwithstanding, this prompts an expansion in AC misfortunes to restrictive levels for high voltage applications where an adequately high obstruction is needed to keep the issue current at a worthy level.

\section{B. Differential learning based time particle swarm optimization}

consider the problem are following in the without loss:

$\operatorname{minf}=f(x)$

s.t XeX

where $\chi \subset \mathrm{X}$ is the set of feasible solutions, $\mathrm{n}=$ dimensionality search space, $d . H$. the no. of decision variables that are the behavior to be learned in context of the social learning

The big picture illustrating above process is shown in Figure 2. 


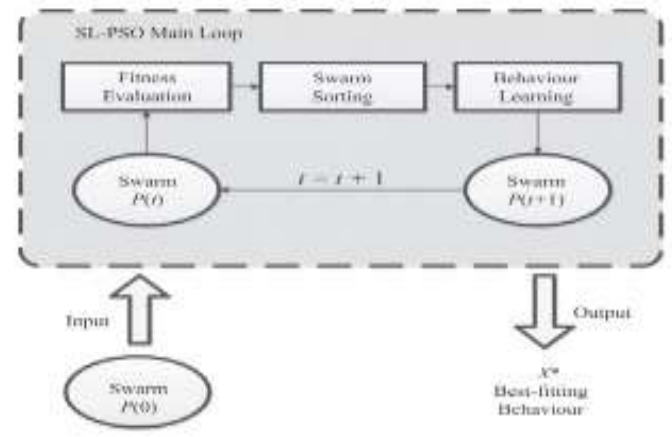

Fig. 2 The proposed SL-PSO of main component

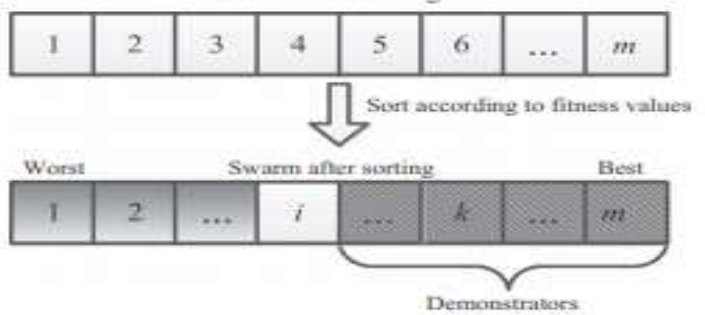

Fig. 3 behavior learning in DL-PSO and the Swarm sorting.

Subsequently, molecule I might gain from various demonstrators in the current multitude. Since this segment is propelled from the impersonation conduct in regular social learning, it is meant as impersonation segment. Similarly, molecule I doesn't gain from gbest either; all things considered, it gains from the aggregate conduct of the entire multitude, i.e., the mean conduct of all particles in the current multitude, denoted by $\bar{X}_{J}(t)$, where $\bar{X}_{J}(t)=\frac{\sum_{1}^{m} X_{i}^{j}}{m}$. Since this segment actuates a multitude level congruity, it is signified as the social impact segment, and correspondingly, the control boundary is meant as the social impact factor. For straightforwardness, the three control boundaries in old style PSO $(\omega, \mathrm{c} 1$ and $\mathrm{c} 2)$ have been supplanted with three irregular coefficients $r 1(t), r 2(t)$ and $r 3(t)$, which will be arbitrarily created inside $[0,1]$ once the refreshing methodology is performed.

\section{Converter system designing for local loads}

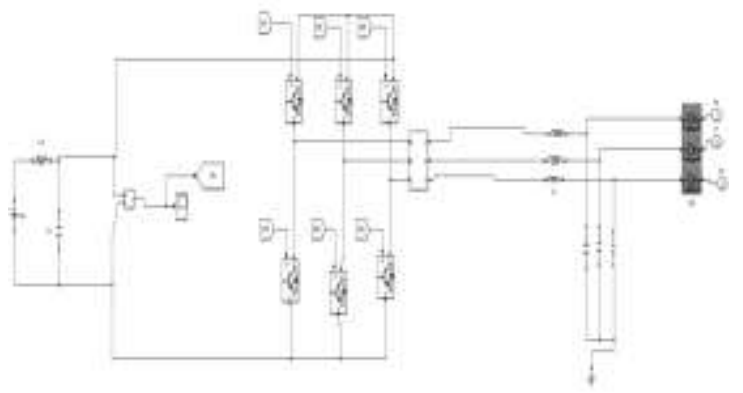

Fig. 4 Converter system implementation in MATLAB/SIMULINK

The inverter control planning has been done as such as to further develop the framework boundaries. The planning has been done in $\mathrm{dq} 0$ reference edge to facilitate the investigation of the essential parts and their individual changes. The framework persistently keeps a beware of the variable boundaries and updates according to the necessity.

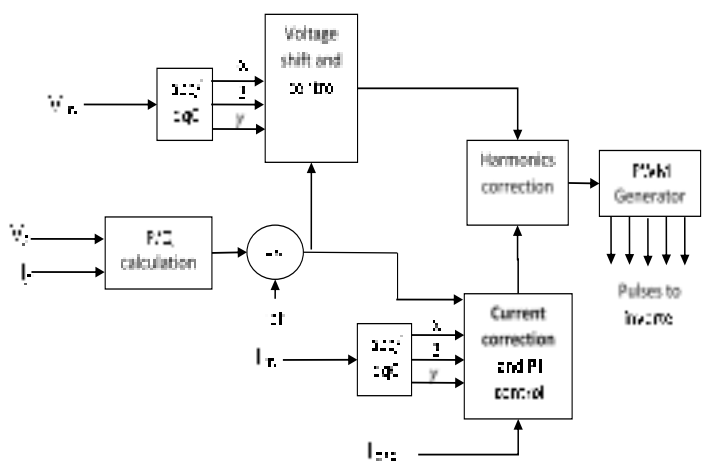

Fig. 5: SPWM with dq reference controller

The above regulator supplies pulse to the inverter, which is a three-leg 6-pulse inverter. The regulator acknowledges lattice boundaries, load boundaries and inverter yield boundaries as info. The dynamic and responsive force prerequisites have been checked and should be improved as changes happen. The guideline of the current setpoint assumes an unequivocal part in controlling the responsive force by managing the stage and the heap interest by directing the addition boundary of the PI guideline. Harmonic correction occurs before the pulse generation signal is sent to the PWM generator. This controller is designed to work and be updated at every change point in the system to demonstrate better pulse and thus achieve improved output parameters.

\section{RESUlts}

The study of the work discusses following case:

CASE 1: Low voltage line subjected to LLLG fault.

CASE 2: Low voltage line subjected to LLG fault.

CASE 3: Low voltage line subjected to LG fault.

The hand-off based SFCL-MATLAB/SIMULINK model deals with the transfer circuit reaction and SFCL power dissemination when the mistake happens. The proposed framework with AIbased control of the hand-off to identify the flaw current is relied upon to give the best of outcome, that is, the dispersal of the current and the rebuilding of ordinary activity in a brief timeframe when the framework is wound down Instability. In each of the three cases, the four frameworks are examined: 
System 1: Low voltage line fault analysis without SFCL.

System 2: Low voltage line fault analysis with SFCL and Relay circuit but no AI based operation control.

System 3: Low voltage line fault analysis with SFCL and Relay circuit with DL_PSO technique.

System 4: Low voltage line fault analysis with SFCL and Relay circuit with ACO technique.

\section{A. CASE 1: Low voltage line subjected to LLLG fault}

In the principal case, the test framework is free of any SFCL gadget and any kind of calculation. The framework transfer is enacted after a high current stream is distinguished in the circuit during the issue condition. The electrical switch disengages the heap from the mains. The framework is analyzed to confirm its capacity to naturally address the mistake.

The bends of voltage yield and current yield are examined in the accompanying figures.

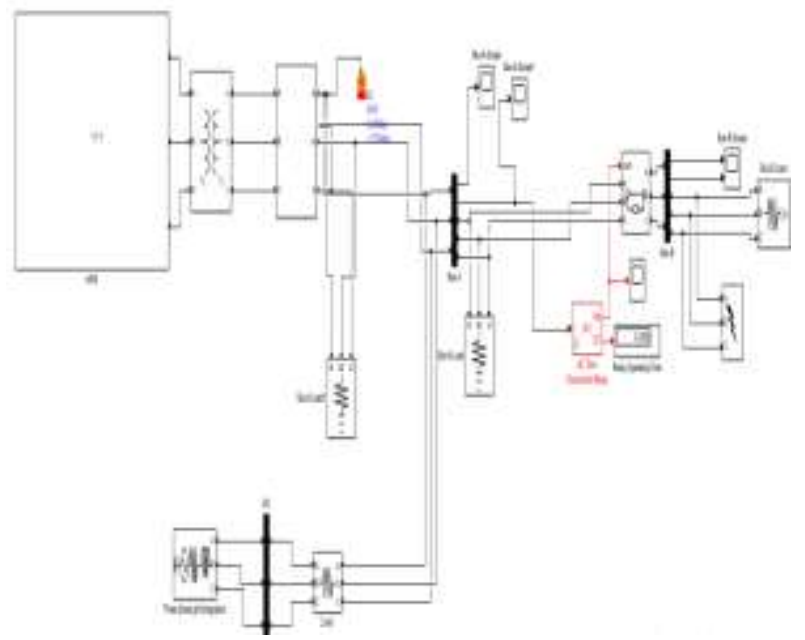

Fig. 6 line fault analysis in system 1 without SFCL.

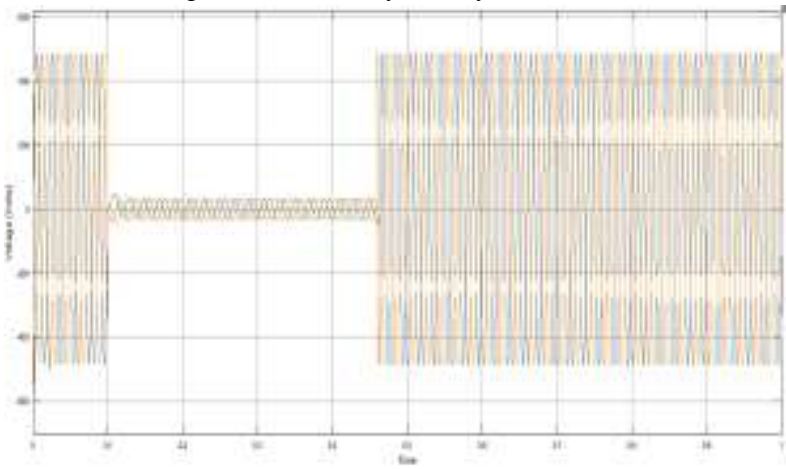

Fig. 7 Voltage output from the system 1 having no SFCL subjected to LLLG fault

When the fault occurs is the reduce voltage is reduced from $500 \mathrm{~V}-0$ volts zero. This completely isolates the power from the system. To solve this problem, we used SFCL in the following models.

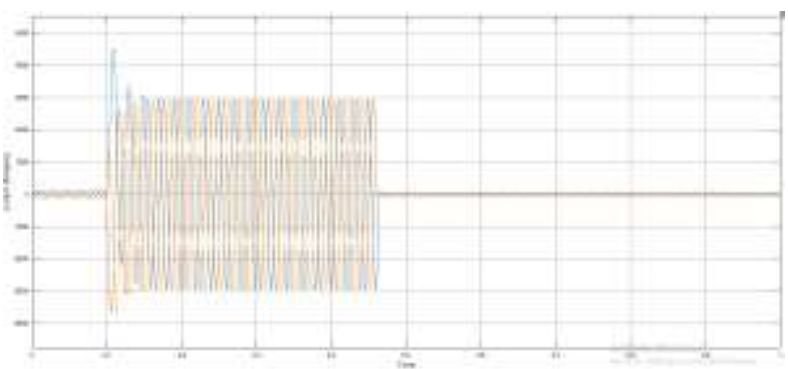

Fig. 8 Current o/p from system 1 having no SFCL subjected to LLLG fault The framework's capacity to fix the bug has worked on over the test framework where SFCL isn't utilized. With this gadget, be that as it may, an unexpected interference of the mains force can be kept away from. To contemplate the impact of SFCL in a framework, the voltage yield and current yield waveforms are dissected below,

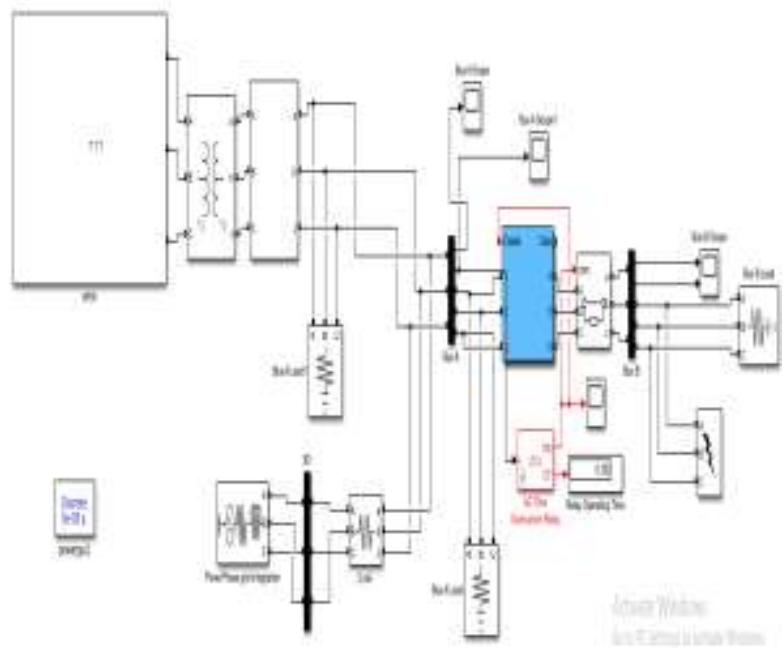

Fig. 9 System 2 Comprising of SFCL in local loads.

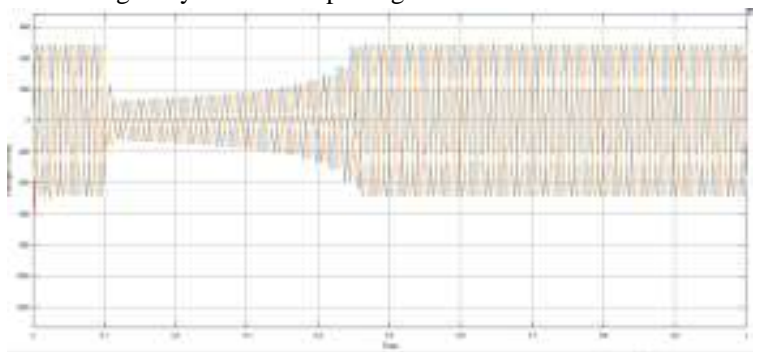

Fig. 10 Voltage o/p from system 2 having SFCL (AI technique no use) subjected to LLLG fault

The figure shows the framework yield voltage when no AI-based innovation is utilized for the transfer however SFCL is utilized in the circuit. At the point when the shortcoming happens, the voltage esteem drops strongly, however starts to recuperate in an extremely brief time frame in light of the fact that during this time the current in Rsh is redirected from SFCL. As the issue current is decreased in 0.352 seconds, the framework will continue ordinary activity. 


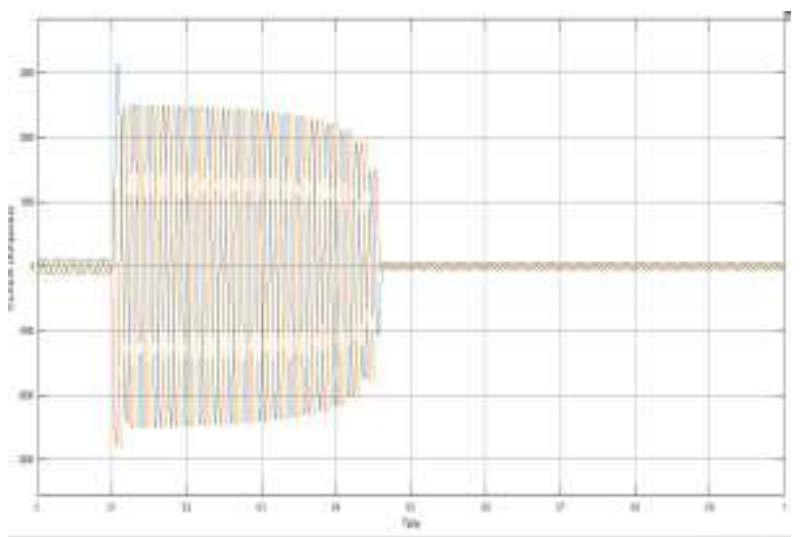

Fig. 11 Current output from the system 1 having SFCL (no AI technique) subjected to LLLG fault

The figure shows the waveform of the current output in the system with SFCL but without the AI-based control technology for relay circuit coordination control. It is observed that when the fault occurs, the current rises to a high value of 2500 amperes in 0.1 seconds.

This large current then passes through the Rsh of the SFCL and hence its value reductions. After a working season of 0.352 seconds, the framework at long last continues ordinary activity and the ebb and flow stretches around 80 amps once more.

From this model it is presumed that the working season of the test framework on the off chance that 1 and that of the test framework on the off chance that to are something similar. Rather than continually staying at 0 , the voltage got back to a specific worth in SFCL because of the deficiency of fault current.

The voltage ought to recuperate from the deficiency quicker than in the past case, which is produced at 0.1 seconds when the framework is reproduced for 1 second.

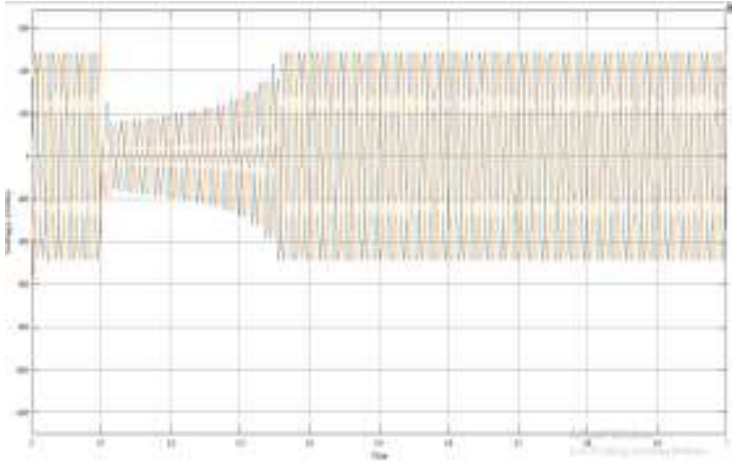

Fig. 12 Voltage output from the system 3 having SFCL with DL_PSO subjected to LLLG fault

The fig. 12 shows the yield voltage of the transfer framework, which is controlled with the molecule swarm enhancement method for its coordination control, and furthermore works on the affectability of flaw current location. It was found that the use of SFCL does not reduce the voltage to zero during the fault current and improves the transient stability of the system and since it is recover from the fault in the $0.215 \mathrm{sec}$.

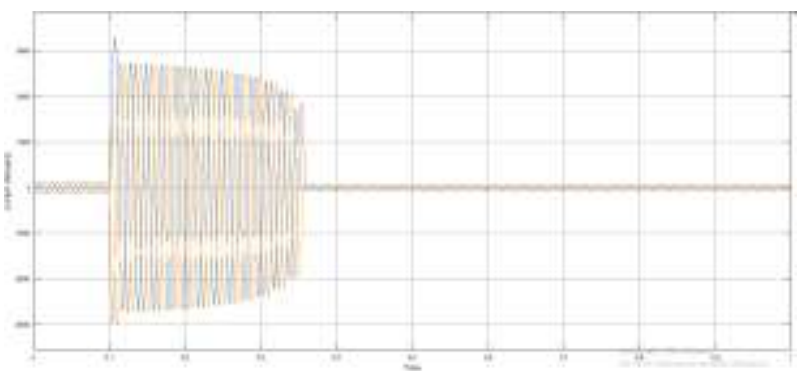

Fig. 13 Current o/p from system 3 having SFCL with DL_PSO subjected to

$$
\text { LLLG fault }
$$

The figure shows the current version of the PSO-based control system. Electricity prices have been found to go up to $3000 \mathrm{~A}$ peak, then $2500 \mathrm{~A}$ at 0.1 seconds when the fault is generated. However, the current dissipation occurs in the SFCL and the framework returns to the normal operation within a short period of 0.215 seconds.

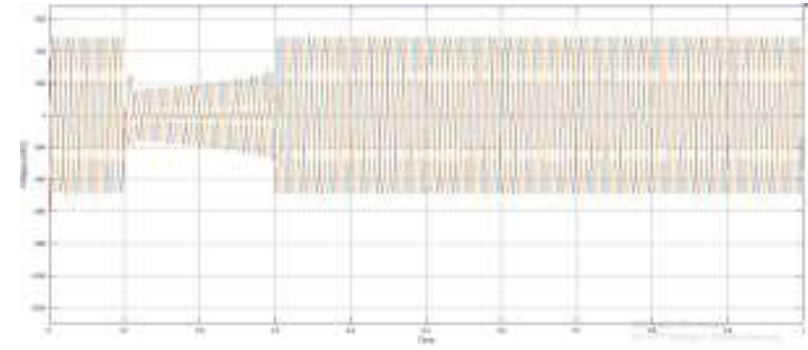

Fig. 14 Voltage output from the system 4 having SFCL with ACO subjected to LLLG fault

The figure above shows the output voltage of the proposed ACO technique for the relay operations. The framework voltage is biased to 0.1 seconds when the fault is generated and begins to slowly increase as the current in the SFCL is diverted. With this technique, if the whole system works for 1 second, the whole process happens a small interval of the $0.197 \mathrm{sec}$.. The error is generated in three stages.

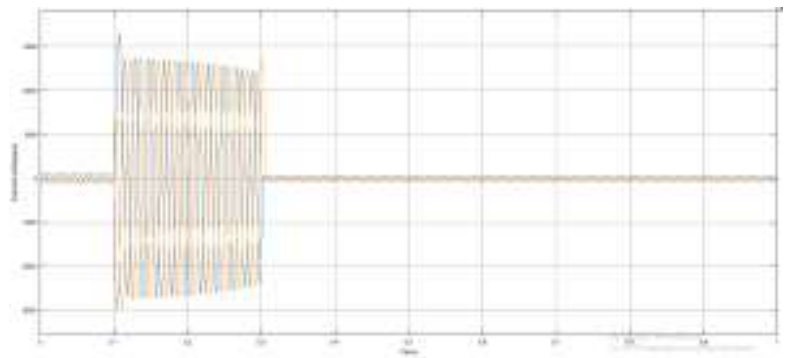

Fig. 15 Current o/p from system 4 having SFCL with ACO subjected to LLLG fault

B. CASE 2: Low voltage line subjected to LLG fault. 


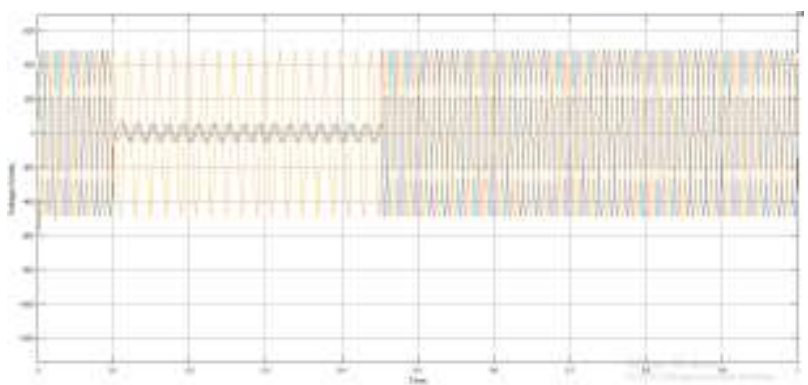

Fig. 16 Voltage o/p from system 1 having no SFCL subjected to LLG fault

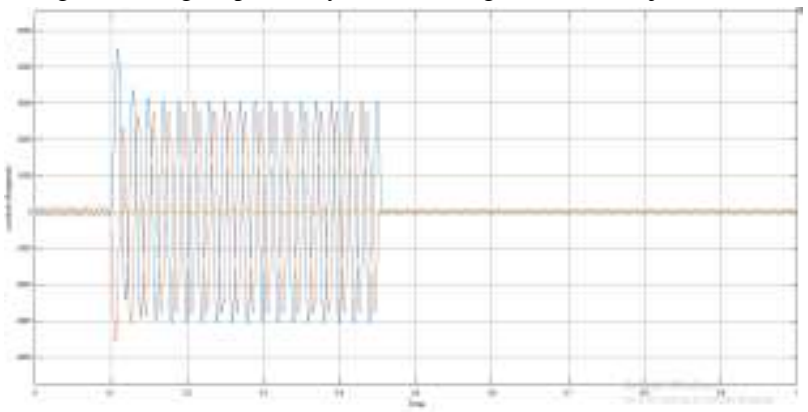

Fig. 17 Current o/p from system 1 having no SFCL subjected to LLG fault

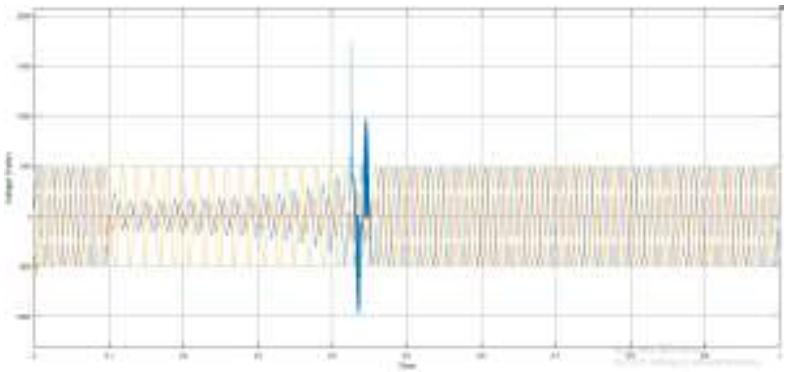

Fig. 18 Voltage o/p from system 2 having SFCL (AI technique no use) subjected to LLG fault

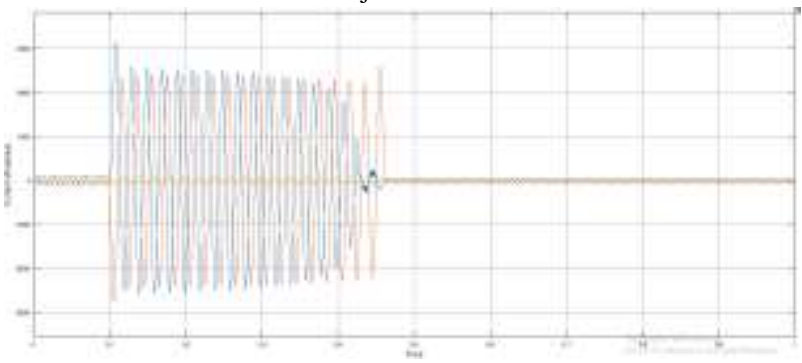

Fig. 19 Current o/p from system 2 having SFCL (no AI technique) subjected to LLG fault

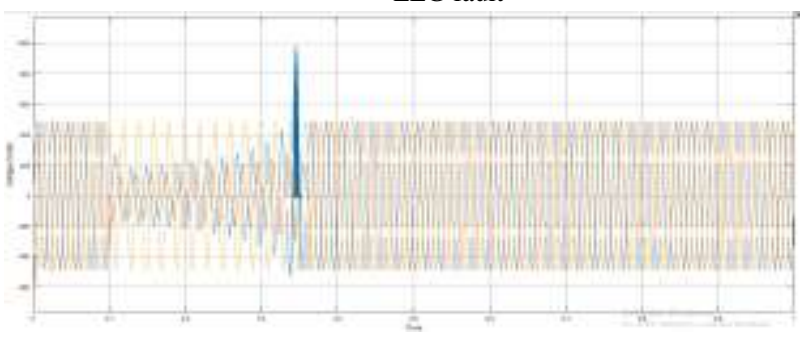

Fig. 20 Voltage o/p from system 3 having SFCL by DL_PSO subjected to LLG fault

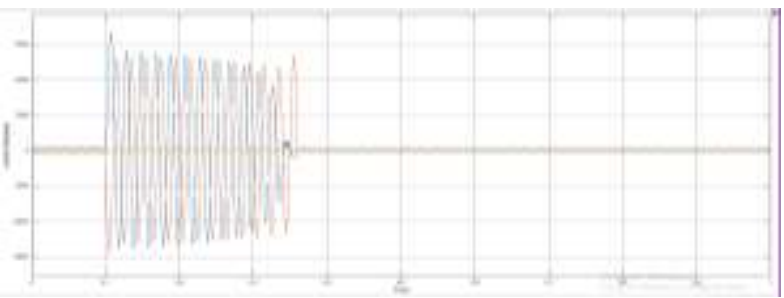

Fig. 21 Current o/p from system 3having SFCL with DL_PSO subjected to LLG fault

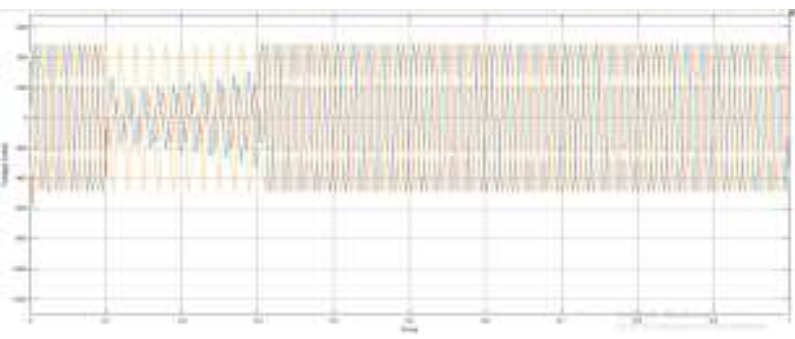

Fig. 22 Voltage o/p from system 4 having SFCL with ACO subjected to LLG fault

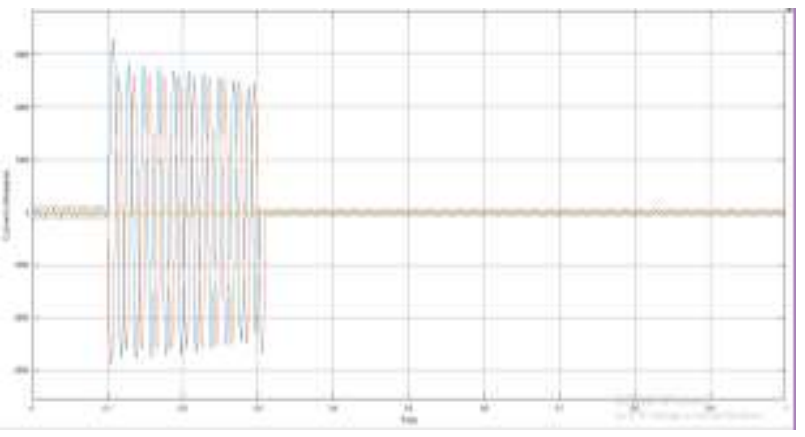

Fig. 23 Current o/p from system 4 having SFCL with ACO subjected to LLG fault

C. CASE 3: Low voltage line subjected to LG fault.

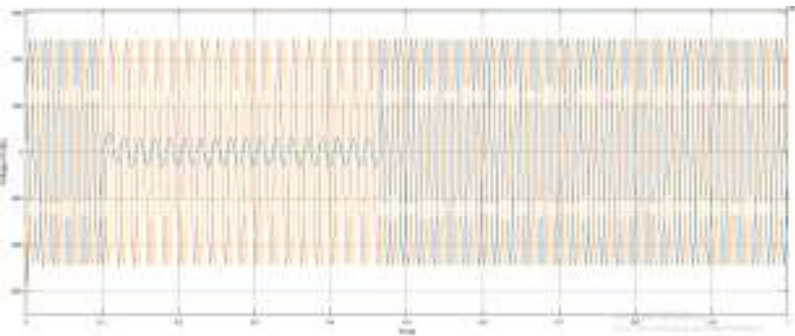

Fig. 24 Voltage o/p from system 1 having no SFCL subjected to LG fault

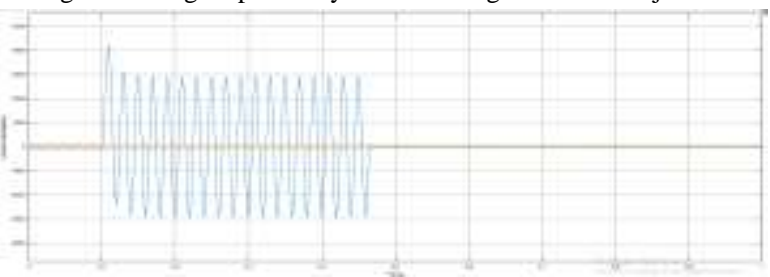

Fig. 25 Current o/p from system 1 having no SFCL subjected to LG fault The value of current in the two phases of system 1 having no SFCL remains high approximately 3000A for the entire duration of fault which is 0.3561 seconds 


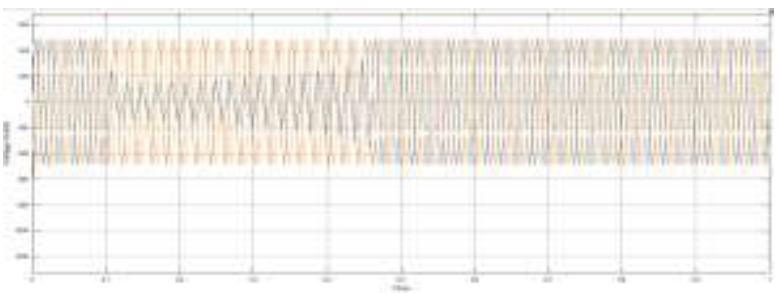

Fig. 26 Voltage o/p from system 2 having SFCL (no AI technique) subjected to LG fault

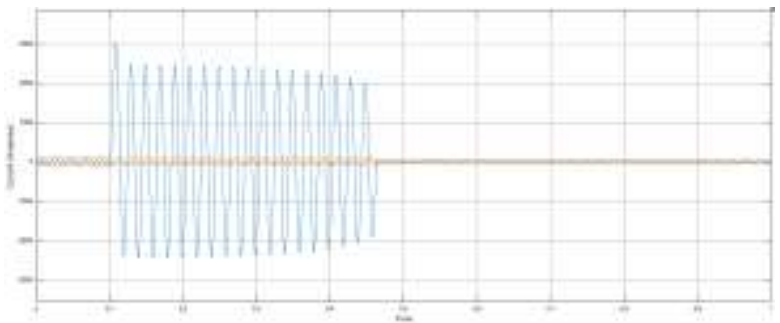

Fig. 27 Current o/p from system 2 having SFCL (no AI technique) subjected to LG fault

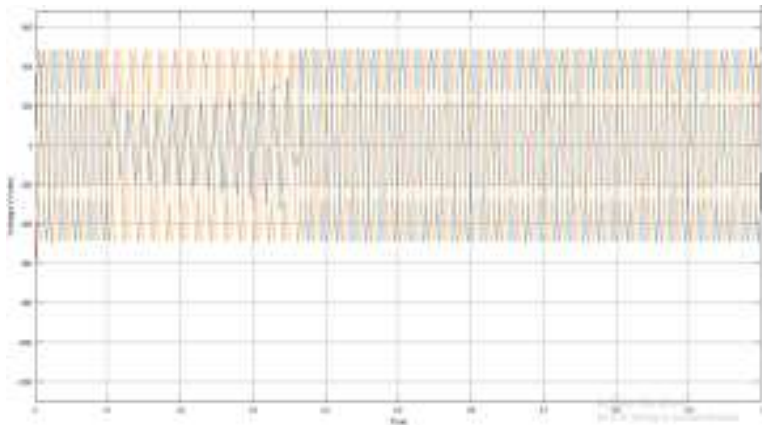

Fig. 28 Voltage o/p from system 3 having SFCL with DL_PSO subjected to LG

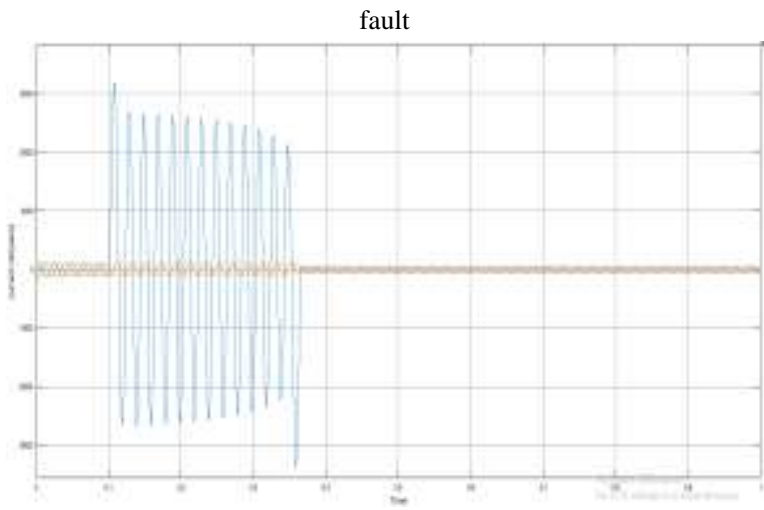

Fig. 29 Current o/p from system 3 having SFCL with DL_PSO subjected to LG fault

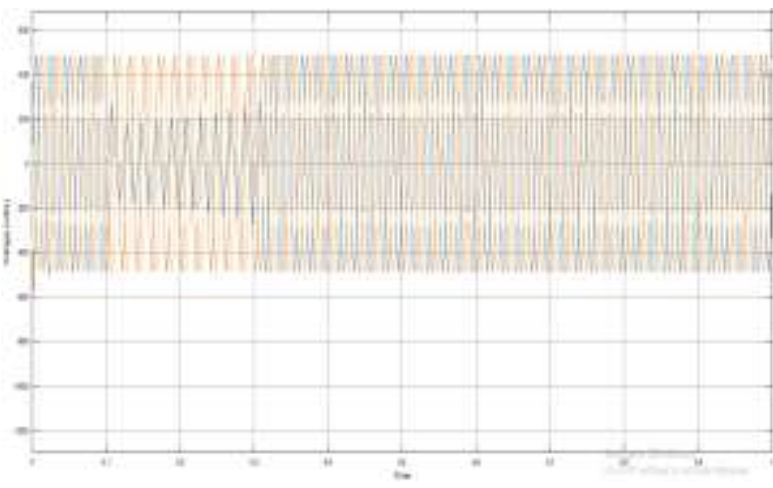

Fig. 30 Voltage o/p from system 4 having SFCL with ACO subjected to LG fault

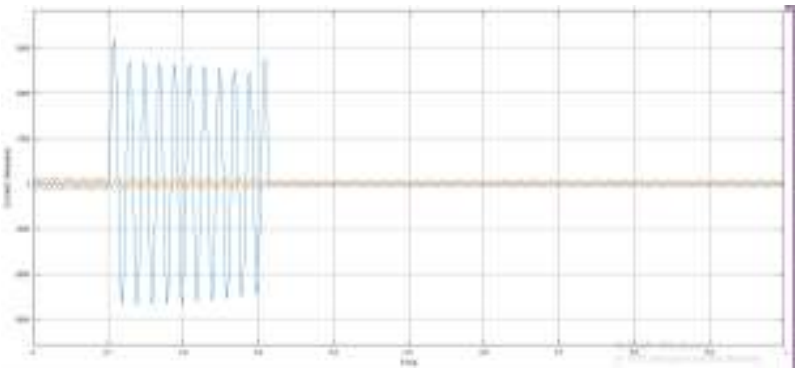

Fig. 31 Current o/p from system 4 having SFCL with ACO subjected to LG fault

\begin{tabular}{|c|c|c|c|c|}
\hline $\begin{array}{l}\text { Type of } \\
\text { fault }\end{array}$ & $\begin{array}{l}\text { Time in } \\
\text { System } 1 \\
\text { (seconds) }\end{array}$ & $\begin{array}{l}\text { Time in } \\
\text { System } 2 \\
\text { (seconds) }\end{array}$ & $\begin{array}{l}\text { Time in } \\
\text { System } 3 \\
\text { (seconds) }\end{array}$ & $\begin{array}{l}\text { Time in } \\
\text { System } 4 \\
\text { (seconds) }\end{array}$ \\
\hline LLLG & 0.3526 & 0.352 & 0.215 & 0.197 \\
\hline LLG & 0.3462 & 0.3499 & 0.2007 & 0.2006 \\
\hline LG & 0.3561 & 0.358 & 0.2099 & 0.2017 \\
\hline
\end{tabular}

\section{CONCLUSION}

The residual current levels of a composite power grid have generally increased due to the growing demand for electricity. This expansion in issue current, if not satisfactorily weakened, can surpass the most extreme upsides of the board. Superconducting fault Current Limiter (SFCL) is an adaptable option in contrast to utilizing regular defensive gadgets because of its effective alternatives for decreasing remaining current in the primary lingering current cycle, low weight and zero impedance during ordinary activity.

Here we offer resistive SFCLs if an issue happens in a basic low voltage (LV) organization. To survey the effect of SFCL in the electrical framework under investigation, a period area approach is utilized to assess the short out flow in the electrical framework and counterfeit control methods are proposed to accomplish the objective. 
The work prompted investigates a test framework and analyzed the impacts of SFCL on it by changing control calculations for quick identification and lessening of flaw flows in the hand-off circuit. The examination was performed for two fundamental calculations, to be specific SFCL Relay Operation with Time Particle Swarm Optimization Based on Differential Learning (DL_PSO) and Adaptive Ant Colony Optimization (ACO). Relay operation control based on ant colonies has proven to be the most effective for all types of faults in the low voltage line.

The results conclude that the proposed optimization control based on A-ACO is effective for the activity of the transfers as per the restricting circuit and the shortcoming current analyzer. The second model of the crossover framework reasons that the framework with lingering current restricting circuit and analyzer lessens the increment in leftover current and keeps the voltage from dropping to nothing, regardless of whether no innovation dependent on man-made brainpower and activity is utilized time stays as before. Man-made intelligence based strategies further lessen the working time, which likewise makes the framework more productive, as the voltage is reestablished to its typical worth in a short measure of time when the test framework is reproduced for 1 second in a MATLAB /SIMULINK. The time taken by the ACO algorithm to restore normal operating conditions in the line was 0.197 seconds, 0.206 seconds and 0.27 seconds for LLLG, LLG and LG errors, respectively.

\section{FUTURE SCOPE}

Obviously, the new kind SFCL with various engineering is as yet under progress. The work can be additionally incorporated with various engineering for SFCL circuit and study its activity in those SFCL. The utilization of quick exchanging, constrained commutated gadget like IGBT shunted with a variable opposition, can work on the presentation of the framework altogether during unusual conditions. Further the upgrade of this plan can prompt a superior yield and can make this framework to work for low voltage ride through LVRT too.

\section{REFERENCE}

[1] Xiaoping, Xue Zhang, Wang, Weiyang Wu, and. Guerrero. "Asymmetrical Grid Fault Ride-Through Strategy of Three-Phase Grid Connected Inverter Considering Network Impedance Impact in LowVoltage Grid.” IEEE, volume 29, issue.03, 2014.

[2] Kaiting LI, Junjie Huare, Tianran LI, Jianfei YANG. "Research on Low Voltage Ride through of the Grid-Connected PV System.” International Conference on Advances in Energy, 2015.

[3] K. Arulkumar, K.Palanisamy, Vijaykumar. "Recent Advances and Control Techniques in Grid Connected PV System-A Review.” IJRER, 2016.

[4] AlwinVinifred Christopher, Ramesh Rengaswamy "Grid Variables Synchronization using Optimization Algorithm for Distributed
Generation System under Grid Fault Conditions" IJRTE, Vol.8 Issue5, 2020.

[5] P. Jyothi Bhaskara Rao and B. Srikanth, "Harmonic Reduction in Grid Connected Solar PV System By Using SVPWM Technique”, Int. J. Appl. Eng.Res., Vol. 11, issue 6 2016, pp. 3853-3858.

[6] N. Kumar, I. Hussain, B.Singh and Panigrahi, "Normal harmonic search algorithm based MPPT of solar PV system," 2016 s(IICPE), 2016, pp. 16.

[7] N. Kumar, I. Hussain, B. Singh and B. K. Panigrahi, "Normal Harmonic Search Algorithm-Based MPPT for Solar PV System and Integrated With Grid Using Reduced Sensor Approach and PNKLMS Algorithm," in IEEE T. Ind. Appl., volume 54, issue 6, pp. 6343-6352, 2018.

[8] C. D. Fuentes, Renaudineau, and S. Bernet, "Power density-driven design methodology of power converters for photovoltaic applications," 2017 ICIT, Toronto, ON, 2017, pp.458-463. 